\title{
Impact of COVID-19 on the outcomes of gastrointestinal surgery
}

\author{
Rahul Gupta $^{1}\left[\right.$ ] Jyoti Gupta ${ }^{2} \cdot$ Houssem Ammar $^{3}$
}

Received: 25 February 2021 / Accepted: 22 April 2021 / Published online: 29 April 2021

(c) Japanese Society of Gastroenterology 2021

\begin{abstract}
COVID-19 pandemic has brought a paradigm shift in the treatment of various surgical gastrointestinal disorders. Given the increasing number of patients requiring hospitalization and intensive care for SARS-CoV-2 infections, various surgical departments worldwide were forced to stop or postpone elective surgeries to save the health resources for COVID-19 patients. Since the declaration of the COVID-19 pandemic by the World Health Organization on 12th March 2020, the recommendations from the surgical societies kept evolving to help the surgeons in making informed decisions regarding patient care. Moreover, various socio-economic and epidemiological factors have come into play while deciding the optimal approach towards patients requiring gastrointestinal surgery. Surgeries for many abdominal diseases such as acute appendicitis and acute calculous cholecystitis were postponed. Elective surgeries were triaged based on the urgency of performing the surgical procedure, the hospital burden of COVID-19 patients, and the availability of healthcare resources. Various measures were adopted such as preoperative screening for SARS-CoV-2 infection, use of personal protective equipment, and the COVID19-free surgical pathway to prevent perioperative SARS-CoV-2 transmission. In this article, we have reviewed the recent studies reporting the outcomes of various gastrointestinal surgeries in the COVID-19 pandemic era and the recommendations from various surgical societies on the safety precautions to be followed during gastrointestinal surgery.
\end{abstract}

Keywords SARS-CoV-2 $\cdot$ COVID-19 $\cdot$ Liver transplant $\cdot$ Bariatric surgery $\cdot$ Colorectal cancer

\section{Introduction}

Since the first declaration of the COVID-19 outbreak in Wuhan, China, in December 2019, the disease has spread rapidly across the globe affecting more than 96 million individuals and led to more than 2 million deaths in 13 months [1]. The exponential surge in the number of patients affected by COVID-19 has led to an unprecedented crisis in health care facilities. Limited health care resources such as personal protective equipment, intensive care facilities forced the surgical departments throughout the globe to re-schedule their surgeries by postponing elective cases and performing only emergency life-saving procedures [1-3]. At the beginning of 2020, there were reports of postoperative COVID-19

Rahul Gupta

rahul.g.85@gmail.com

1 Department of Gastrointestinal Surgery, Synergy Institute of Medical Sciences, Dehradun 248001, Uttarakhand, India

2 Department of Radiation Oncology, Swami Rama Himalayan University, Dehradun, India

3 Department of Surgery, Sousse Hospital, Sousse, Tunisia after elective surgeries with a lack of evidence on its impact on surgical outcomes $[4,5]$. Hence, many surgical societies recommended postponing elective surgeries until the availability of health care resources and reduction in the burden of COVID-19 cases [6, 7].

To facilitate evidence-based decision-making in the COVID-19 pandemic era, we have performed a detailed literature review and summarized the findings of studies reporting the impact of SARS-Cov-2 infection on the outcomes of gastrointestinal surgeries. Besides, we have provided an overview of the recommended surgical guidelines on the perioperative safety measures to prevent transmission of SARS-CoV-2 infection among the patients and health care workers and optimize the outcomes of abdominal surgeries.

\section{Impact of COVID-19 pandemic on gastrointestinal surgical services}

All the surgical services have been severely affected during this ongoing COVID-19 pandemic. In a study published in May 2020, it was estimated that during the 12 
weeks of peak disruption due to the COVID-19 pandemic, about 28.4 million surgeries would be canceled worldwide, which would require 45 weeks to clear the backlog of surgeries if the surgical centers increase their routine surgical volume by $20 \%$ after the recovery from the pandemic [8]. However, this estimate is far from reality as the pandemic is still not over. Elective surgeries are still being postponed at several centers worldwide to take care of the COVID patients with limited health resources. In the United Kingdom, nearly all elective bariatric surgeries were postponed in April 2020, the peak COVID-19 pandemic [9]. Various other abdominal surgeries, including colorectal surgeries $[10,11]$, solid organ transplants [12], were severely affected in 2020.

In a Spanish study comparing acute care surgeries performed before and during the COVID pandemic, the authors reported significantly fewer laparoscopic surgeries in the pandemic era (43.3\% vs. $63.6 \%, p=0.001)$ [13]. The reasons were fear of spreading SARS-CoV-2 infection with laparoscopic aerosols and fewer surgeries performed for acute cholecystitis during the pandemic era. The authors reported about a 59\% decrease in the number of emergency surgeries during the pandemic. The time from onset of symptoms to patient arrival to the emergency department was longer ( $44.6 \mathrm{~h}$ vs. $71 \mathrm{~h}, p<0.001$ ). The surgeries for bowel obstruction and ventral hernia increased, while that for acute cholecystitis decreased during the pandemic period. An Indian study by Kapoor et al. reported that only 314 gastrointestinal surgeries were performed from March to July 2020 compared to 914 surgeries during the same time in the previous year [14].

At the beginning of the COVID-19 pandemic, laparoscopy was believed to increase the risk of viral transmission based on the previous reports of viral aerosolization (HPV, HBV, HIV) due to the chimney effect of the smoke that escaped from the trocars [15]. Hence, many surgical societies recommended restricted use of laparoscopy and diathermy during the pandemic [16]. However, with accumulation of experience and clinical data analysis, it was found that there was no significant perioperative SARS-CoV-2 infection transmission among the patients and healthcare workers with laparoscopy $[15,17,18]$. Hence, the new recommendation was to perform laparoscopic surgeries using appropriate precautions such as personal protective equipments, smoke evacuation devices, if it was clearly beneficial to the patients and reduced their hospital stay $[19,20]$. The CLOUD-19 collaborative group conducted an online survey in Italy and found that the proportion of surgeons performing less than 20\% laparoscopic elective surgeries during the three phases (March-May, June-September, October-December 2020) of COVID-19 pandemic was $25.8 \%$, $12.4 \%$ and $19.3 \%$, respectively [21]. These findings indicated that the practice of laparoscopy in COVID-19 pandemic continues to remain restricted despite lack of evidence of SARS-CoV-2 transmission by surgical smoke.

COVID-19 pandemic has caused significant delay in the diagnosis and treatment of various gastrointestinal cancers [22-27]. The decline in number of surgeries in 2020 has been due to multiple factors including delayed diagnosis due to national lockdowns, hesitance of patients to visit hospital, cancelation of endoscopic activity and other cancer screening programs, and cancelations/postponement of elective surgeries due to shortage of hospital resources [25, 27, 28]. A study from United Kingdom (UK) reported that all nonemergent endoscopies were stopped for 6 weeks in March and April 2020 due to COVID-19 pandemic leading to significant reductions in diagnostic endoscopies and decreased the number of new cancer cases from 677 per week in the pre-COVID period to 283 per week in the COVID times [28]. Another UK study had estimated that about 5\% and $16 \%$ increase in deaths is likely to occur from esophageal and colorectal cancer due to delay in diagnosis and therapeutic interventions [22]. According to a study by Sud et al., delay in cancer surgery by more than 3 months and 6 months may lead to $>17 \%$ and $>30 \%$ reduction in the survival of patients with stage 2 or 3 gastrointestinal cancers [26]. An international survey involving 76 centers across the world found that $50 \%$ curative and/or palliative treatments for liver cancer and $44 \%$ liver transplants were canceled during COVID-19 pandemic from March to June 2020 [25].

\section{Impact of preoperative COVID-19 on the postoperative outcomes}

Various studies have been published in the literature to report the outcomes of gastrointestinal surgery in the COVID-19 pandemic. In this review article, we have summarized the findings of key original articles and meta-analysis which can help the clinicians in day-to-day practice (Table 1).

\section{Emergency surgery}

An initial study from Wuhan, China comparing outcomes of eight COVID-19 patients with 22 non-COVID patients requiring emergency abdominal surgery found no significant impact of COVID-19 on the postoperative outcomes, namely, the blood parameters, respiratory support, and mortality [29]. The authors concluded that patients with acute abdomen should be offered surgical treatment if required, irrespective of the COVID-19 status. In another study from Wuhan, the authors compared outcomes of six patients with preoperative COVID-19 pneumonia and 28 patients without SARS-CoV-2 infection who underwent emergency surgery [30]. They reported a higher duration of hospital stay in COVID patients but no difference in the postoperative 


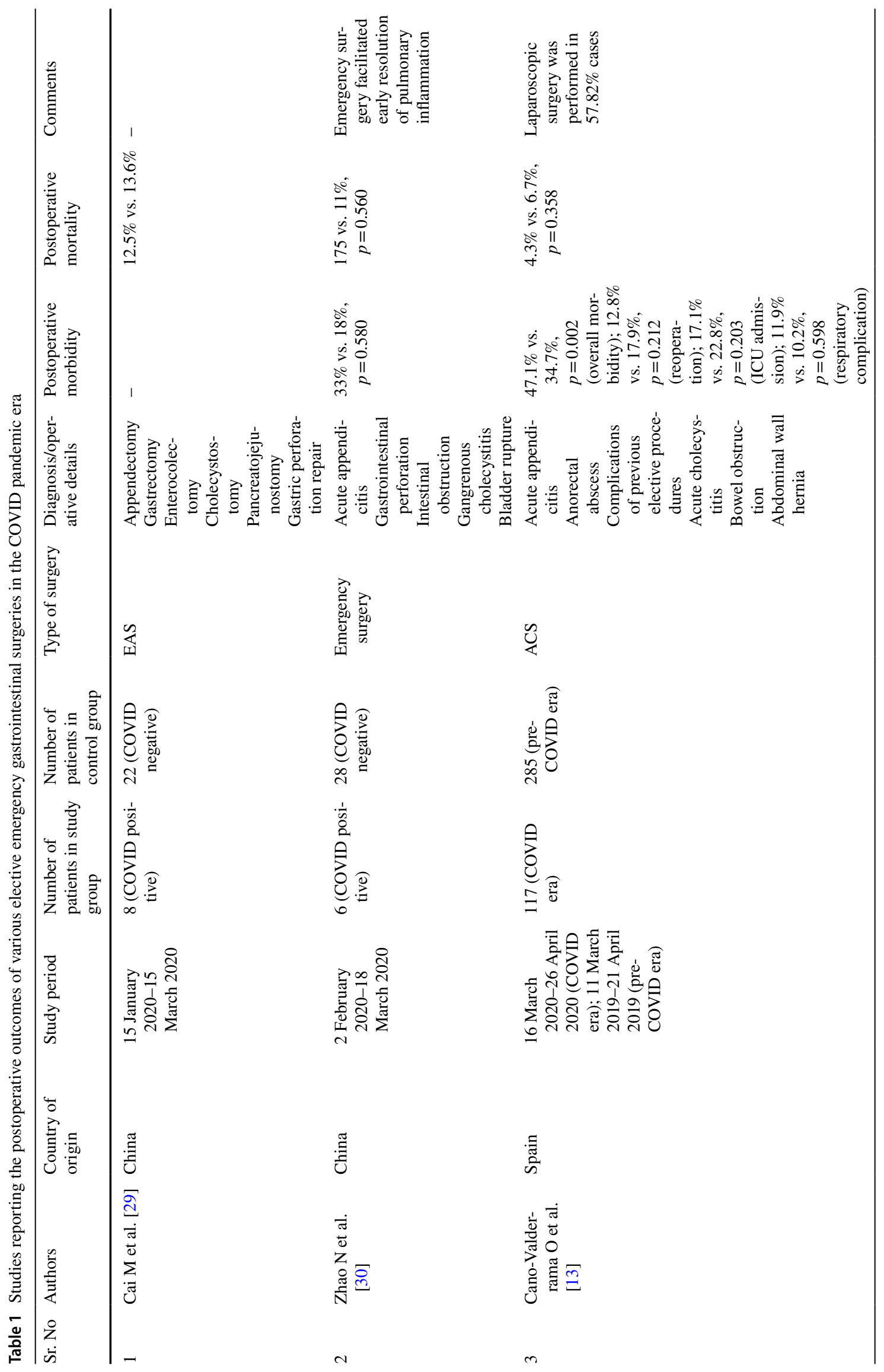


Clinical Journal of Gastroenterology (2021) 14:932-946

935

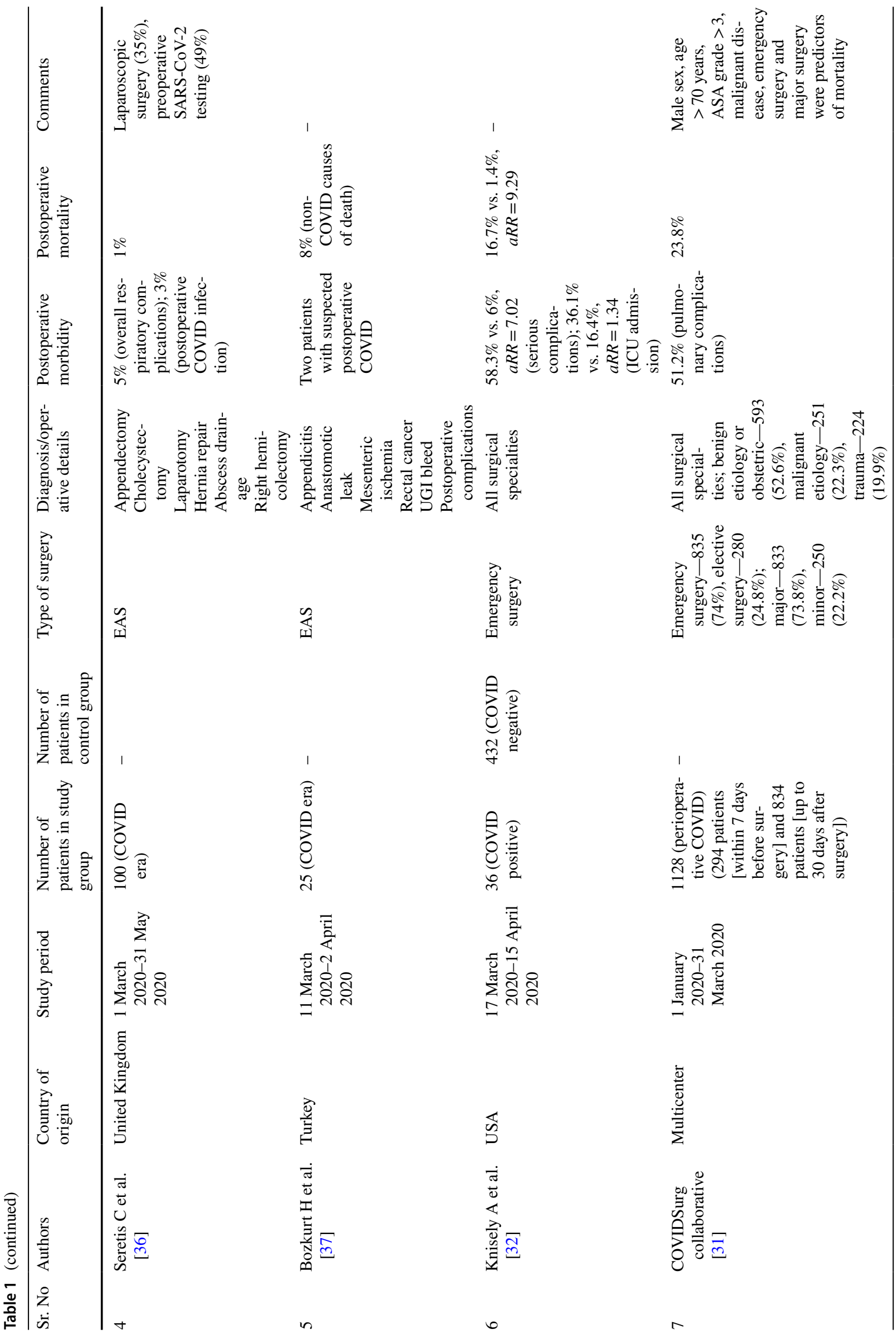

Springer 


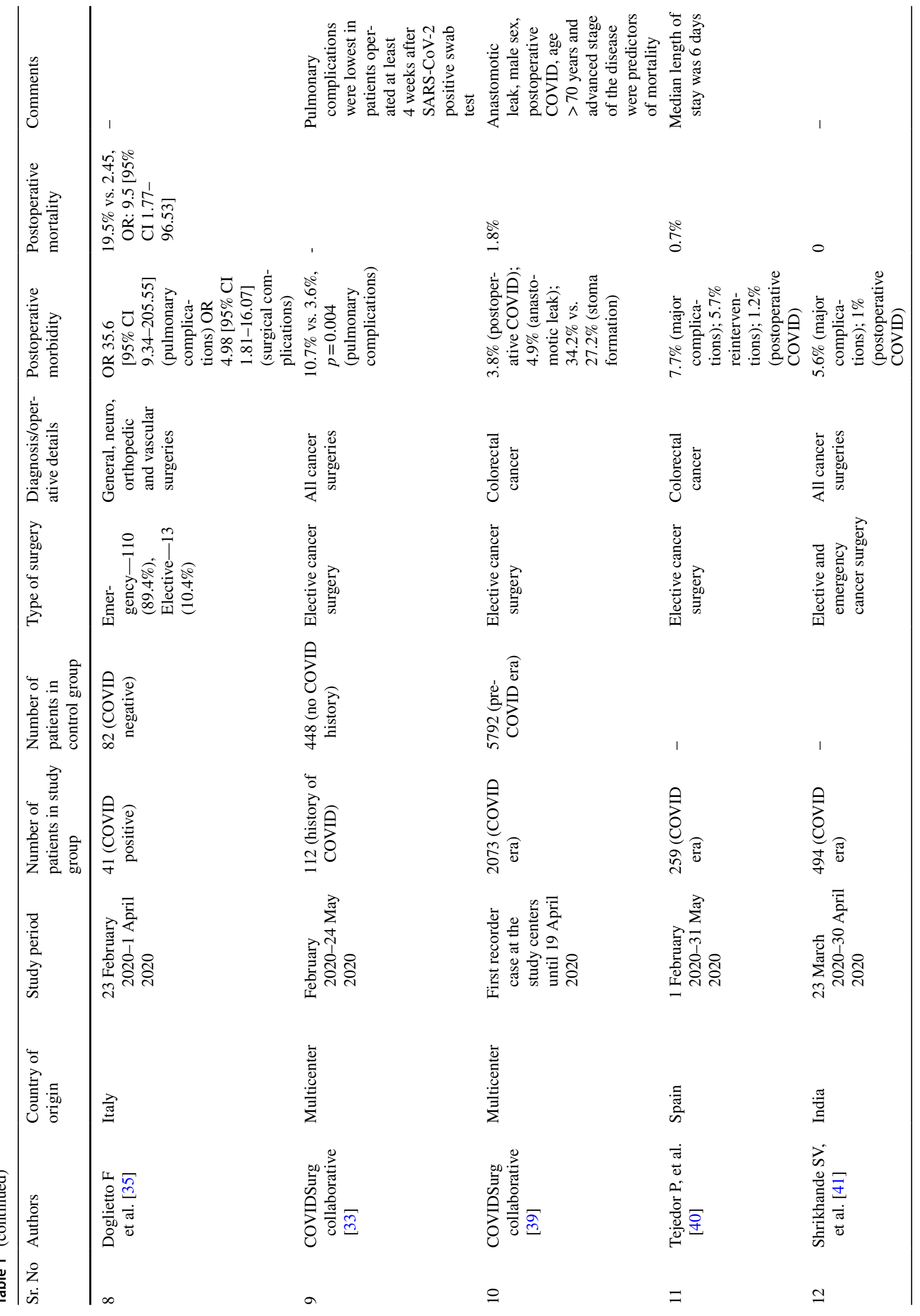




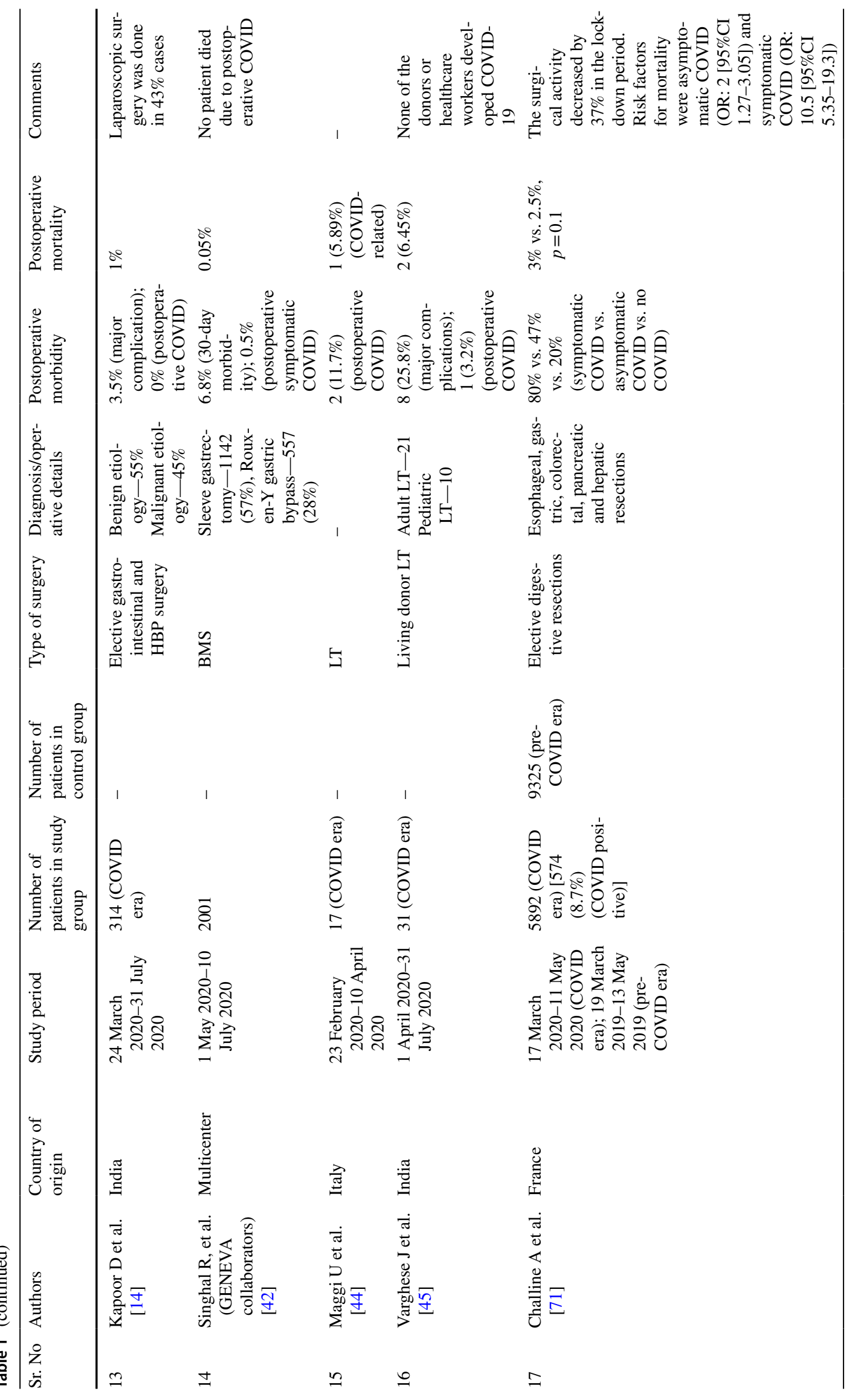




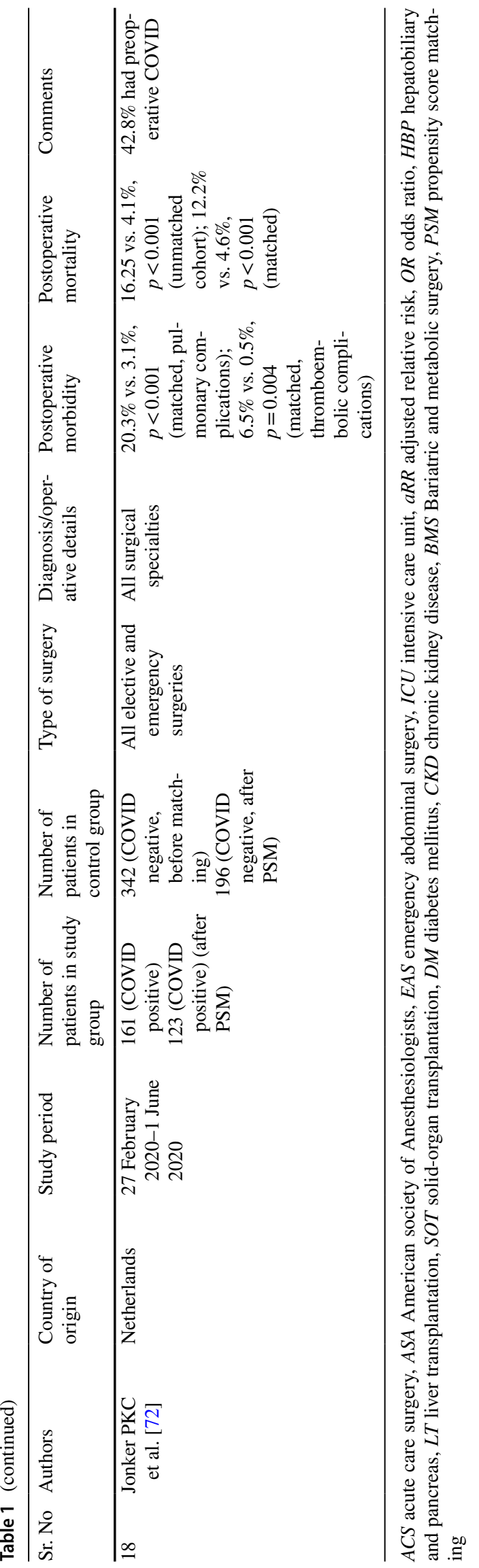

complications or mortality between the two groups. The authors found that emergency surgery helped treat the surgical pathology and benefitted in the early resolution of the pulmonary inflammation [30]. So, it was recommended that emergency surgery should be conducted, if required, with all the safety precautions to prevent SARS-CoV-2 transmission among other patients and medical staff members.

A Spanish study compared outcomes of emergency abdominal surgeries in 285 patients from the pre-COVID era with 117 patients during the COVID pandemic [13]. Out of the 117 patients in the COVID era, four patients were suspected, and three patients had confirmed SARS-CoV-2 infection. The overall morbidity rate was higher $(47.1 \%$ vs. $34.7 \%, p=0.002)$ while the reoperation rate $(12.8 \%$ vs. $17.9 \%, p=0.212)$ and mortality rate $(4.3 \%$ vs. $6.7 \%$, $p=0.358)$ was similar in the two groups [13].

In an international multicenter study conducted by COVIDSurg Collaborative involving 1128 surgical patients with perioperative SARS-CoV-2 infection [preoperative (within 7 days before surgery) in 294 patients and postoperative (up to 30 days after surgery) in 834 patients], the pulmonary complications and mortality were reported in $51.2 \%$ and $23.7 \%$ cases, respectively [31]. The factors associated with 30-day mortality included male sex, age $>70$ years, American Society of Anesthesiologists (ASA) grade $>3$, malignant disease, emergency surgery, and major surgery. Hence, the authors concluded that the threshold to perform surgery in patients with the risk factors mentioned above should be high [31].

In an American study of 468 patients undergoing emergency surgery, 36 patients had confirmed COVID-19 [32]. The perioperative serious complications rate $[58.3 \%$ vs. $6 \%$, adjusted relative risk $(a R R)=7.02$ ], ICU admission rate $(36.1 \%$ vs. $16.4 \%, a R R=1.34)$ and mortality rates $(16.7 \%$ vs. $1.4 \%, a R R=9.29)$ were significantly higher in patients with COVID-19 [32].

Hence, SARS-CoV-2-infected patients requiring emergency surgery should be offered surgical treatment and strict protocol should be followed to prevent viral transmission to the health workers and other patients.

\section{Elective surgery}

A multicenter comparative study of patients with $(n=112)$ and without $(n=448)$ history of SARS-CoV-2 infection undergoing elective cancer surgery was conducted [33]. The study found that previous SARS-CoV-2 infection was associated with an increased risk of postoperative pulmonary complications $(10.7 \%$ vs. $3.6 \%$, $p=0.004)$. The authors also reported that the risk of pulmonary complications and mortality was lowest when the surgery was performed at least 4 weeks after the positive swab test in those with SARS-CoV-2 infection [33]. A 
subsequent large-scale study by the COVIDSurg collaborative involving 140,231 patients from 116 countries found that surgeries performed more than 7 weeks after the diagnosis of SARS-CoV-2 infection was associated with similar mortality risk as that of those without history of SARS-CoV-2 infection [34]. They reported that the 30-day mortality in patients without SARS-CoV-2 infection was $1.5 \%$ while in those with SARS-CoV-2 infection was $4.1 \%, 3.9 \%, 3.6 \%$ and $1.5 \%$ in patients having surgery within 0-2 weeks, 3-4 weeks, 5-6 weeks and $\geq 7$ weeks, respectively [34].

In an Italian cohort study involving 41 COVID-19 patients matched with 82 non-COVID-19 patients who underwent surgical treatment (general, neurosurgery, orthopedic and vascular surgeries), the surgical complications were significantly higher in the COVID group (Odds ratio 4.98; 95\% CI 1.81-16.07) [35]. Moreover, the 30 -day mortality rate in the COVID-19 group was also significantly higher (Odds ratio 9.5; 95\% CI 1.77-96.53).

Hence, in patients with SARS-CoV-2 infection, nonemergent surgeries should be postponed for 4-7 weeks and non-surgical procedures should be considered in such patients, if feasible.

\section{Impact of COVID-19 pandemic on the postoperative outcomes of gastrointestinal surgery}

\section{Emergency surgery}

A retrospective analysis of 100 patients who underwent emergency abdominal surgery in the United Kingdom from March to May 2020 found that the incidence of overall respiratory complications, postoperative COVID-19 infection rate, and the mortality rate was 5\%,3\% and $1 \%$, respectively [36]. There was no additional morbidity or mortality associated with COVID-19 infection.

In a Turkish study, 25 patients underwent emergency abdominal surgery from March to April 2020 in the COVID-19 era [37]. None of the patients had preoperative COVID-19, and two had suspected COVID-19. The authors concluded that emergency surgeries could be safely performed in COVID pandemic hospitals if adequate precautions are taken.

Hence, it is recommended that emergency surgeries should be performed during the COVID-19 pandemic. Preoperative SARS-CoV-2 testing should be done prior to surgery. If the patient is not infected, then the COVIDfree surgical pathway should be adopted to prevent perioperative SARS-CoV-2 transmission to the patient [38].

\section{Elective surgery for gastrointestinal cancer}

In the largest multicenter cohort study conducted by COVIDSurg collaborative group, 2073 patients without preoperative COVID from 40 countries who underwent elective colorectal surgery for cancer were included [39]. The postoperative SARS-CoV-2 infection rate was $3.8 \%$ cases, the anastomotic leak rate was $4.9 \%$, and the 30 -day mortality rate was $1.8 \%$. The independent risk factors for mortality were an anastomotic leak, male sex, postoperative SARSCoV-2 infection, age $>70$ years, and advanced stage of the disease. Compared with the pre-COVID data of 5792 patients from 54 countries published in 2015 and 2017, the anastomotic leak rate and hospital stay were lesser, but the mortality rate was higher. Additionally, the stoma formation rate was higher in the study than in the pre-COVID era (34.2\% vs. $27.2 \%)$ [39].

A multicenter Spanish study included 259 patients who underwent surgery for colorectal cancer during the COVID19 pandemic [40]. The major complications, reinterventions, and postoperative COVID-19 related pneumonia were $7.7 \%$, $5.7 \%$, and $1.2 \%$ cases, respectively. The median length of hospital stay was 6 days, and the mortality rate was $0.7 \%$.

In an Indian study by Shrikhande et al. on the outcomes of major cancer surgery during the COVID-19 pandemic, 494 patients were included, 84 (17\%) patients underwent surgery for gastrointestinal cancer [41]. The authors reported a low incidence of major complications and zero mortality. Also, only six out of 494 patients developed postoperative SARS-CoV-2 infection.

Kapoor et al. reported the outcomes of 314 gastrointestinal surgeries performed during the COVID-19 pandemic (March to July 2020) [14]. Among these, 144 operations were performed for various gastrointestinal cancers. The authors compared the outcomes of these surgeries with that of the previous year during the same time (March to July 2019). Significantly fewer surgeries were being performed during the pandemic, with a higher proportion of cancer surgeries than the previous year $(44.9 \%$ vs. $23.5 \%, p<0.001)$. However, no significant difference in the incidence of major complications and 30-day mortality were observed in the two time periods [14].

Thus, elective gastrointestinal surgeries can be performed in the COVID-19 pandemic provided the healthcare resources are available and all the safety precautions are maintained to prevent perioperative transmission of SARSCoV-2 infection among the patients and health care workers.

\section{Bariatric and metabolic surgery (BMS)}

An international multicenter cohort study (GENEVA) was conducted to determine the outcomes of bariatric surgery in the COVID-19 era [42]. In this study, the 30-day outcomes 
of 2001 adult patients ( $>18$ years) from 127 hospitals in 35 countries who underwent BMS between 1st May and 10th July 2020 were analyzed. The most BMS procedures performed were sleeve gastrectomy [1142 (57\%)], Roux-en-Y gastric bypass [557 (28\%)], and mini-gastric bypass [215 $(11 \%)]$. The 30 -day morbidity and mortality rates were $6.8 \%$ $(n=138)$ and $0.05 \%(n=1)$, respectively. The postoperative symptomatic COVID infection rate was $0.5 \%(n=10)$. None of the patients who developed COVID required ICU care and none died [42].

A nationwide French study of more than 4 million obese individuals found that 8286 individuals required admission for COVID-19 between January and May 2020. Among these COVID-19 obese patients, 541 patients had a history of BMS. Interestingly, on logistic regression analysis, the authors found that previous BMS was associated with a significantly reduced risk of mechanical ventilation and mortality [43].

Based on these reports, it can be concluded that BMS can be offered to the obese patients if the healthcare resources are present and appropriate measures are taken to prevent perioperative transmission of SARS-CoV-2 infection. In areas with high incidence of COVID-19, BMS should be resumed once there is reduction in the number of COVID19-related hospital admissions.

\section{Solid-organ transplantation (SOT)}

All the patients undergoing SOT receive immunosuppression, making them prone to develop opportunistic infections. As there was no evidence on the incidence, severity, and impact of SARS-CoV-2 infection in immunocompromised patients at the beginning of the COVID-19 pandemic, SOT was performed only as a life-saving procedure in a limited number of patients. Nevertheless, with the availability of health care resources and a better understanding of COVID19, several transplant centers have resumed SOT. Most of the transplant centers followed strict perioperative protocol such as preoperative COVID-19 testing of the recipient and donor, COVID free surgical pathway, maintaining isolation in the postoperative period, and COVID-19 testing of the caregivers (Table 2).

A prospective study from Northern Italy included 17 patients who received liver transplantation (LT) between February and April 2020 [44]. Out of these 17 patients, two patients developed COVID-19 infection after LT, and one patient died due to COVID on postoperative day 30 . The authors concluded that LT is safe in the COVID-19 pandemic, and LT programs can be continued if the required healthcare resources are available [44].

In a multicenter Indian study involving 31 living donor LT patients (21 adults and 10 pediatric patients) from April to July 2020, one patient developed SARS-CoV-2 infection
46 days after surgery and was recovering at the time of publication [45]. In this patient, mycophenolate mofetil was stopped, and the doses of tacrolimus and steroids were modified. No antivirals were administered due to the risk of hepatitis. None of the donors or transplant team members developed SARS-CoV-2 infection during the study period. The authors emphasized the importance of maintaining strict protocol in preventing the development of COVID-19 in the recipients, donors, or transplant team members [45].

The waitlist mortality rate in the liver transplant candidates is $5 \%$ at 6 months, $10.4 \%$ at 1 year and $19 \%$ at 3 years [46]. However, the mortality in patients with high Model for End-stage Liver Disease (MELD) score $>30$ is more than $50 \%$ at 3 months [47]. While, the success rate of LT is $90 \%$ at 1 year and $80 \%$ at 3 years [48]. As the benefit outweighs the risk, considering the ongoing COVID-19 pandemic, most of the transplant societies recommend that the decision to perform LT should be taken based on the local incidence of SARS-CoV-2 infection, availability of resources, severity of the liver disease in the waitlist candidates and the regional/national health policies $[49,50]$. In addition, each candidate must be explained the additional risk of SARSCoV-2 infection and its complications after LT during the counseling process.

\section{Sars-Cov-2 infection in liver transplant recipients}

The nationwide study from the Italian registry reported that the cumulative incidence of COVID infection in SOT recipients was $1.02 \%$, which was significantly higher than the non-transplant patients $(0.4 \%)(p<0.05)$ [51]. Among 450 SOT recipients with SAR-CoV-2 infection, 89 (19.8\%) were LT recipients). The cumulative incidence of SAR$\mathrm{CoV}-2$ infection was lower in LT recipients compared to kidney and heart recipients. More than $80 \%$ of SOT recipients had undergone transplants more than 1 year before the SAR-CoV-2 infection. Out of 450 patients, 123 patients died during the follow-up period. The 30-day and 60-day mortality rates were lower in LT recipients compared to heart and kidney recipients (Table 3) [51].

A study by Kates et al. involving data from more than 50 transplant centers in the United States reported outcomes of 482 SOT recipients with laboratory-confirmed SARCoV-2 infection [52]. Out of these, 73 (15.1\%) patients had undergone LT. The median age of the included patients was 58 years. The median time post-transplant was 5 years. $376(78 \%)$ patients required hospitalization, including 147 (39\%) patients requiring ICU care and 77 (20.5\%) patients who died within 1 month after the diagnosis of COVID19 (Table 3) [52]. 


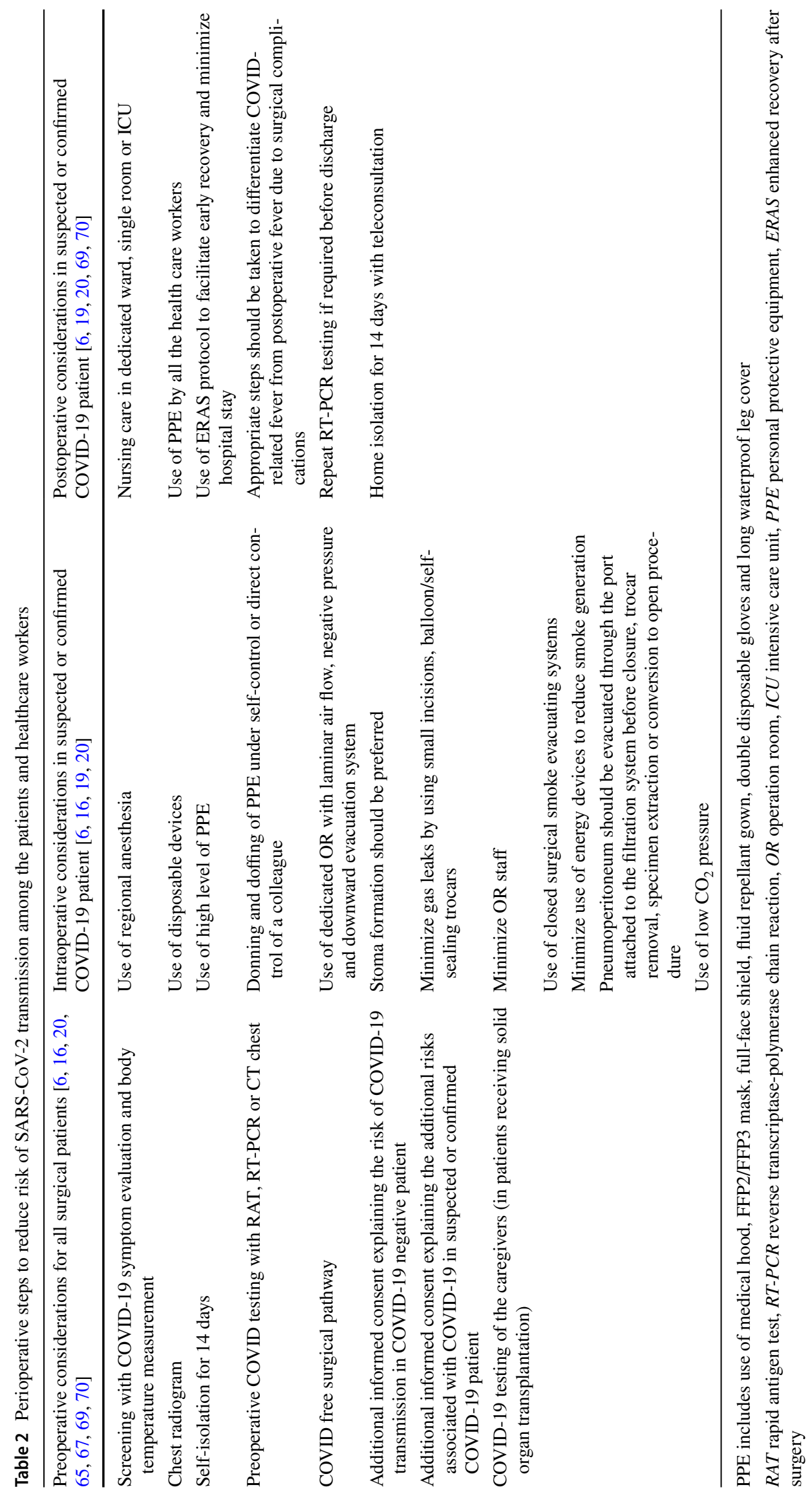




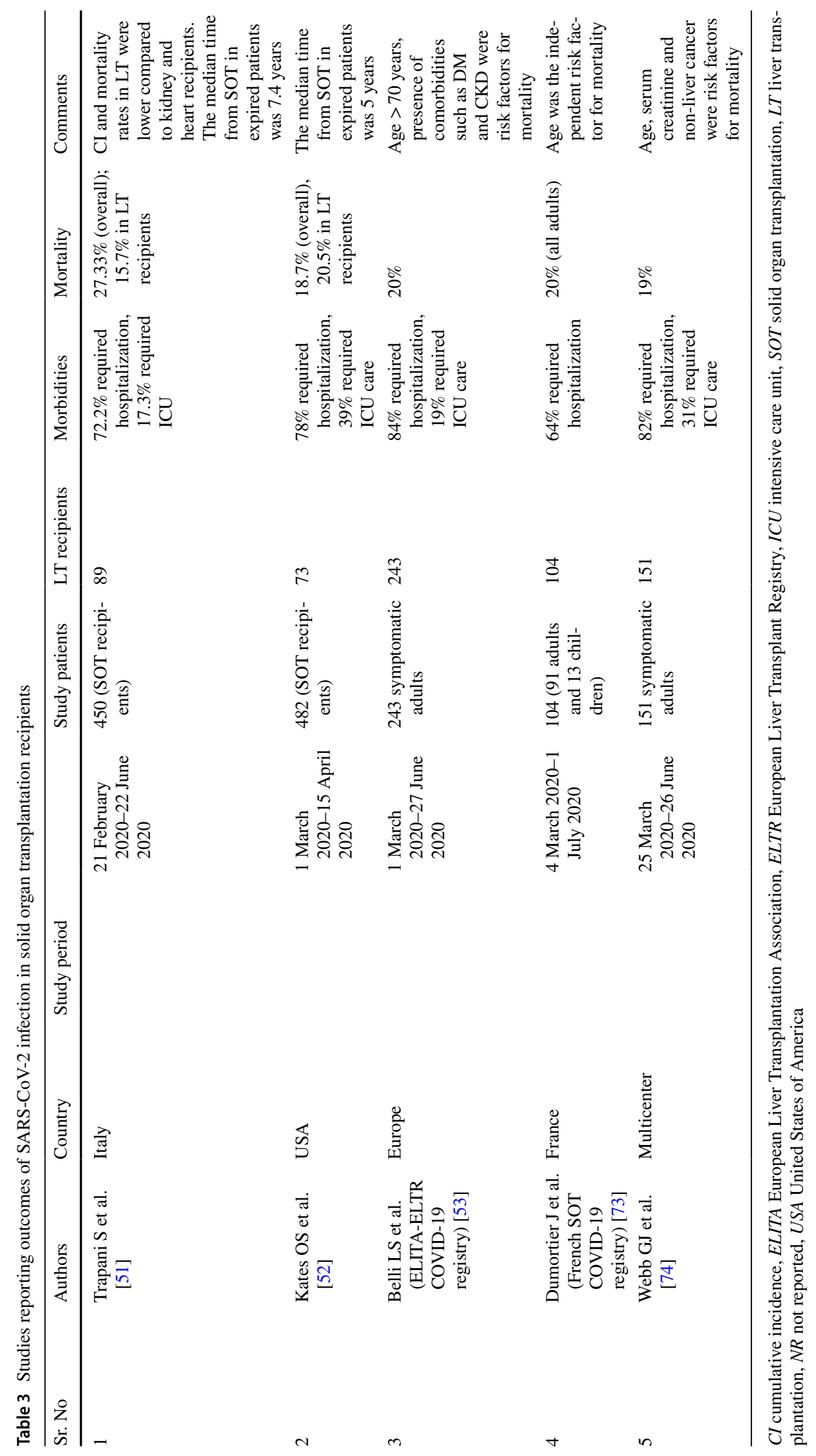


The multicenter study from the European LT Registry (ELTR) is the largest study reporting the outcomes of SARCoV-2 infection in 243 adult symptomatic LT patients from 36 centers [53]. They found that $84 \%$ of patients required hospitalization, including ICU admission in 19\% of cases. About 20\% of LT patients died due to COVID-9, with respiratory failure being the primary cause of death. On multivariate analysis, the authors found that age $>70$ years, comorbidities such as diabetes mellitus, and chronic kidney disease were independent risk factors for mortality (Table 3) [53].

In a comprehensive review and meta-analysis by Raja et al. including 1500 kidney, 505 liver, and 282 other organ transplant recipients, the pooled incidence of hospital admission, ICU admission, mechanical ventilation, and all-cause mortality was $81 \%, 29.3 \%, 25.9 \%$, and $18.6 \%$, respectively [54]. Among the LT recipients, the all-cause mortality rate was $11.8 \%$.

\section{Use of immunosuppression and drugs for COVID infection among LT recipients}

In the ELTR study, the authors reported the use of hydroxychloroquine, lopinavir-ritonavir, low molecular weight heparin, high doses of corticosteroids, and tocilizumab for the SARS-CoV-2 infection in LT recipients [53]. Surprisingly, the use of tacrolimus was associated with a significantly reduced risk of mortality in LT recipients with COVID19 infection [53]. On subgroup analysis, the authors found that patients receiving tacrolimus were younger, had fewer comorbidities, and were less frequently treated with ACE or ARB inhibitors. However, tacrolimus was protective even after taking care of the cofounders. The possible reasons behind the beneficial role of tacrolimus could be direct inhibition of the viral replication and cytokine storm [33]. However, in the multicenter US study by Kates et al., the authors found no association between immunosuppression and 28-day mortality [52].

In the meta-analysis by Raja et al. involving 2772 SOT recipients, the authors reported that immunosuppressive medications, especially antimetabolites, were reduced in $76.2 \%$ of patients, and calcineurin inhibitors were reduced in $38.7 \%$ of patients [54]. Steroids were seldom reduced. The most commonly used drugs to treat COVID-19 were hydroxychloroquine, azithromycin, IL-6 antagonists such as tocilizumab, protease inhibitors, and high-dose steroids, with the pooled incidence of their use ranging from 14.9 to $59.5 \%$ [54].

According to American Association for the Study of Liver Diseases (AASLD) guidelines, the dose of immunosuppressants should not be reduced in LT recipients without COVID-19 [55]. In LT recipients with COVID-19, a reduction in the dose of azathioprine, mycophenolate, and high-dose prednisolone should be considered. The dose of calcineurin inhibitors can be reduced if required but should not be stopped. The use of hydroxychloroquine, azithromycin, and lopinavir-ritonavir to treat COVID-19 is not recommended [55].

\section{Triage of patients for elective surgery during the COVID-19 pandemic}

During the pandemic, triage of patients is required to schedule the elective surgery based on the hospital burden of the COVID-19 patients and the available healthcare resources [56]. To facilitate rational, individualized decision-making, a multidisciplinary team that includes oncologists, surgeons, gastroenterologists, radiologists, and other health care providers can be formed to conduct weekly virtual meetings to discuss the pending elective cases [57]. Priority for surgery is given to cancer patients who have completed neoadjuvant therapy, patients with aggressive cancers for which other therapies are not very effective, patients awaiting the second part of the staged procedures, diagnostic procedures, and patients with acute symptoms such as bleeding, bowel obstruction and bowel perforation [57].

Different triage systems have been proposed to guide the clinicians in decision-making [7, 16, 58-60]. Some triage systems are predominantly based on the proportion of the COVID-19-related hospital admissions and availability of hospital resources [58-60]. At the same time, other triage systems are mainly based on the disease and patient factors to determine the urgency of performing the surgical procedure [7, 60-62]. The multidisciplinary team can use these triage systems to propose an individualized treatment plan for the patients awaiting elective surgeries during the COVID-19 pandemic.

Regarding LT, elective deceased donor LT should be performed only if both donor and recipient are COVID negative and the recipient is from the same city to avoid air travel [63]. Living donor LT should be carried out only for urgent cases as it exposes the donor to the risk of SARS-CoV-2 infection during hospital admission.

\section{Strategies to reduce perioperative transmission of SARS-CoV-2}

In the early period of the COVID-19 pandemic, due to the shortage of personal protective equipment (PPE) and fear of perioperative SARS-CoV-2 transmission to the patients and health care workers, only emergency surgeries were carried in the COVID-19 affected countries. Subsequently, with the availability of PPE, increased healthcare facilities, and a better understanding of the COVID-19 disease, elective surgeries were resumed. However, it is prudent to follow 
the strategies recommended by surgical societies based on the available evidence to prevent perioperative COVID-19 transmission $[6,7,64,65]$. The key recommendations have been summarized in Table $2[66,67]$.

In addition to the general recommendations applicable for all the surgeries, specific precautions need to be followed depending on the type of surgery. For example, in laparoscopy surgeries, extreme care should be taken to prevent SARS-CoV-2 transmission by minimizing aerosol generation, avoiding gas leaks, and appropriate smoke evacuation (Table 2). Surgical smoke is considered to be one of the means of perioperative viral transmission based on the previous reports [21]. The SARS-CoV-2 virus has been found in the peritoneal fluid, bile, ascitic fluid and surgically resected specimens suggesting that the viruses are present in the operative field during the surgery $[18,68]$. But, the evidence to support that SARS-CoV-2 infection is transmitted by surgical smoke is lacking $[15,17,18]$. However, due to the theoretical concerns, it is recommended to use personal protective equipments and smoke evacuation devices, minimize aerosol generation, and avoid gas leaks to prevent perioperative SARS-CoV-2 transmission (Table 2) $[16,19,20]$.

Regarding LT, the recipient should be tested for SARSCoV-2 infection by real-time PCR within $24 \mathrm{~h}$ of LT [69]. All deceased donors should be preferably tested for COVID-19 by bronchoalveolar lavage. Living donors should be tested 24-72 h before LT. Moreover, self or hospitalbased quarantine for 14-21 days before LT should be recommended [70]. If the recipient had active SARS-CoV-2 infection, then LT should be deferred for at least 28 days after symptom resolution and should have two negative RTPCR tests $24 \mathrm{~h}$ apart before LT [69]. The COVID-19-free surgical pathway should be implemented for LT [70]. Postdischarge follow-up hospital visits should be avoided, and online consultation should be encouraged. However, in case of any emergency, the transplant patients should attend the hospital. Treating clinicians should suspect COVID-19 if the recipients develop any COVID-related symptoms such as fever, cough, and breathing difficulty.

\section{Conclusions}

Perioperative SARS-CoV-2 infection has an adverse impact on postoperative outcomes. However, patients with COVID19 should not be denied emergency abdominal surgeries. Elective surgeries should be triaged based on the urgency of performing the surgical procedure, the hospital burden of COVID-19 patients, and the availability of healthcare resources. Routine preoperative screening for SARS-CoV-2 infection should be performed before elective surgeries. Abdominal surgery in COVID patients should be postponed for at least 4 weeks to optimize the postoperative outcomes. All surgeries in COVID-19-negative patients should be performed using the COVID-19-free surgical pathway to prevent perioperative SARS-CoV-2 transmission. LT in COVID-19 pandemic is safe provided it is performed strictly as per the recommended guidelines.

Author contributions RG-Conception of the work, drafting of manuscript, final approval and agreement for the accountability of work. JG-Data collection, drafting of manuscript, final approval and agreement for the accountability of work. HA-Data acquisition, revising of the manuscript, final approval and agreement for the accountability of work

\section{Declarations}

Conflict of interest The authors have no potential conflict of interest to declare.

\section{References}

1. Johns Hopkins University of Medicine. COVID-19 Map. 2020. https://coronavirus.jhu.edu/map.html. Accessed 20 Jan 2021.

2. Bartlett DL, Howe JR, Chang G, et al. Management of cancer surgery cases during the COVID-19 pandemic: considerations. Ann Surg Oncol. 2020;27:1717-20.

3. Manzia TM, Angelico R, Parente A, et al. Global management of a common, underrated surgical task during the COVID-19 pandemic: Gallstone disease-an international survery. Ann Med Surg. 2020;57:95-102.

4. Aminian A, Safari S, Razeghian-Jahromi A, et al. COVID-19 outbreak and surgical practice: unexpected fatality in perioperative period. Ann Surg. 2020;272:e27-9.

5. Aminian A, Kermansaravi M, Azizi S, et al. Bariatric surgical practice during the initial phase of COVID-19 outbreak. Obes Surg. 2020;30:3624-7.

6. Francis N, Dort J, Cho E, et al. SAGES and EAES recommendations for minimally invasive surgery during COVID-19 pandemic. Surg Endosc. 2020;34:2327-31.

7. COVID-19: guidance for triage of non-emergent surgical procedures. https://www.facs.org/covid-19/clinical-guidance/triage. Accessed 5 Apr 2020.

8. COVIDSurg Collaborative. Elective surgery cancellations due to the COVID-19 pandemic: global predictive modelling to inform surgical recovery plans. Br J Surg. 2020;107:1440-9.

9. Moussa O, Zakeri R, Arhi C, et al. Impact of COVID-19 on obesity management services in the United Kingdom (The COMSUK study). Obes Surg. 2020;31:904-8.

10. Nunoo-Mensah JW, Rizk M, Caushaj PF, et al. COVID-19 and the global impact on colorectal practice and surgery. Clin Colorectal Cancer. 2020;19:178-90.e1.

11. Mason SE, Scott AJ, Markar SR, et al. Insights from a global snapshot of the change in elective colorectal practice due to the COVID-19 pandemic. PLoS ONE. 2020;15:e0240397.

12. Boyarsky BJ, Po-Yu Chiang T, Werbel WA, et al. Early impact of COVID-19 on transplant center practices and policies in the United States. Am J Transplant. 2020;20:1809-18.

13. Cano-Valderrama O, Morales X, Ferrigni CJ, et al. Acute care surgery during the COVID-19 pandemic in Spain: changes in 
volume, causes and complications. A multicentre retrospective cohort study. Int J Surg. 2020;80:157-61.

14. Kapoor D, Perwaiz A, Singh A, et al. Elective gastrointestinal surgery in COVID times. Indian J Surg. 2021;83:277-83.

15. Antunes D, Lami M, Chukwudi A, et al. COVID-19 infection risk by open and laparoscopic surgical smoke: a systematic review of the literature. Surgeon. 2021. https://doi.org/10. 1016/j.surge.2021.02.003.

16. ACS, guidelines for triage and management of elective cancer surgery cases during the acute and recovery phases of coronavirus disease 2019 (COVID-19) pandemic. 2020. https://www. facs.org//media/files/covid19/acs_triage_and_management_ elective_cancer_surgery_during_acute_and_recovery_phases. ashx. Accessed 25 Apr 2020.

17. Hadjittofi C, Seraj SS, Uddin A, et al. Laparoscopic vs open surgery during the COVID-19 pandemic: what are the risks? Ann R Coll Surg Engl. 2021. https://doi.org/10.1308/rcsann. 2020.7067.

18. Cheruiyot I, Sehmi P, Ngure B, et al. Laparoscopic surgery during the COVID-19 pandemic: detection of SARS-COV-2 in abdominal tissues, fluids, and surgical smoke. Langenbecks Arch Surg. 2021. https://doi.org/10.1007/s00423-021-02142-8.

19. De Simone B, Chouillard E, Sartelli M, et al. The management of surgical patients in the emergency setting during COVID19 pandemic: the WSES position paper. World J Emerg Surg. $2021 ; 16: 14$.

20. Somashekhar SP, Acharya R, Saklani A, et al. Adaptations and safety modifications to perform safe minimal access surgery (MIS: laparoscopy and robotic) during the covid-19 pandemic: practice modifications expert panel consensus guidelines from academia of minimal access surgical oncology (AMASO). Indian J Surg Oncol. 2020. https://doi.org/10.1007/s13193-020-01254-9.

21. Bracale U, Podda M, Castiglioni S, et al. Changes in surgical behaviors during the COVID-19 pandemic. The SICE CLOUD19 study. Updates Surg. 2021;73:731-44.

22. Maringe C, Spicer J, Morris M, et al. The impact of the COVID19 pandemic on cancer deaths due to delays in diagnosis in England, UK: a national, population-based, modelling study. Lancet Oncol. 2020;21:1023-34.

23. Sud A, Torr B, Jones ME, et al. Effect of delays in the 2-weekwait cancer referral pathway during the COVID-19 pandemic on cancer survival in the UK: a modelling study. Lancet Oncol. 2020;21:1035-44.

24. Morris EJA, Goldacre R, Spata E, et al. Impact of the COVID-19 pandemic on the detection and management of colorectal cancer in England: a population-based study. Lancet Gastroenterol Hepatol. 2021;6:199-208.

25. Muñoz-Martínez S, Sapena V, Forner A, et al. Assessing the impact of COVID-19 on liver cancer management (CERO-19). JHEP Rep. 2021. https://doi.org/10.1016/j.jhepr.2021.100260.

26. Sud A, Jones ME, Broggio J, et al. Collateral damage: the impact on outcomes from cancer surgery of the COVID-19 pandemic. Ann Oncol. 2020;31:1065-74.

27. Rutter MD, Brookes M, Lee TJ, et al. Impact of the COVID19 pandemic on UK endoscopic activity and cancer detection: a National Endoscopy Database Analysis. Gut. 2021;70:537-43.

28. Fernando S, Veli M, Mohammadi B, et al. COVID-19 and its impact on upper gastrointestinal (GI) cancer management. Cancers. 2021;13:397.

29. Cai M, Wang G, Zhang L, et al. Performing abdominal surgery during the COVID-19 epidemic in Wuhan, China: a single-centred, retrospective, observational study. Br J Surg. 2020;107:e183-5.

30. Zhao N, Wu L, Cheng Y, et al. The effect of emergency surgery on acute abdomen patients with COVID-19 pneumonia: a retrospective observational study. Aging. 2020;12:15771-83.
31. COVIDSurg Collaborative. Mortality and pulmonary complications in patients undergoing surgery with perioperative SARS-CoV-2 infection: an international cohort study. Lancet. 2020;396:27-38.

32. Knisely A, Zhou ZN, Wu J, et al. Perioperative morbidity and mortality of patients with COVID-19 who undergo urgent and emergent surgical procedures. Ann Surg. 2021;273:34-40.

33. COVIDSurg Collaborative. Delaying surgery for patients with a previous SARS-CoV-2 infection. Br J Surg. 2020;107:e601-2.

34. COVIDSurg Collaborative. Timing of surgery following SARSCoV-2 infection: an international prospective cohort study. Anaesthesia. 2021. https://doi.org/10.1111/anae.15458.

35. Doglietto F, Vezzoli M, Gheza F, et al. Factors associated with surgical mortality and complications among patients with and without coronavirus disease 2019 (COVID-19) in Italy. JAMA Surg. 2020;155:691-702.

36. Seretis C, Archer L, Lalou L, et al. Minimal impact of COVID-19 outbreak on the postoperative morbidity and mortality following emergency general surgery procedures: results from a 3-month observational period. Med Glas. 2020;17:275-8.

37. Bozkurt H, Gür HÜ, Akıncı M, et al. Evaluation of patients undergoing emergency surgery in a COVID-19 pandemic hospital: a cross-sectional study. Sao Paulo Med J. 2020;138:305-9.

38. Glasbey JC, Nepogodiev D, Simoes JFF, et al. Elective cancer surgery in COVID-19-free surgical pathways during the SARSCoV-2 pandemic: an international, multicenter, comparative cohort study . J Clin Oncol. 2021;39:66-78.

39. COVIDSurg Collaborative, . Outcomes from elective colorectal cancer surgery during the SARS-CoV-2 pandemic. Colorectal Dis. 2021;23:732-49.

40. Tejedor P, Simó V, Arredondo J, et al. The impact Of SARS$\mathrm{CoV}-2$ infection on the surgical management Of colorectal cancer: lessons learned from a multicenter study in Spain. Rev Esp Enferm Dig. 2021;113:85-91.

41. Shrikhande SV, Pai PS, Bhandare MS, et al. Outcomes of elective major cancer surgery during COVID 19 at Tata Memorial Centre: implications for cancer care policy. Ann Surg. 2020;272:e249-52.

42. Singhal R, Tahrani AA, Ludwig C, et al. Global 30-day outcomes after bariatric surgery during the COVID-19 pandemic (GENEVA): an international cohort study. Lancet Diabetes Endocrinol. 2021;9:7-9.

43. Iannelli A, Bouam S, Schneck AS, et al. The impact of previous history of bariatric surgery on outcome of COVID-19. A Nationwide Medico-Administrative French Study. Obes Surg. 2020. https://doi.org/10.1007/s11695-101-05120-z.

44. Maggi U, De Carlis L, Yiu D, et al. The impact of the COVID-19 outbreak on liver transplantation programs in northern Italy. Am J Transplant. 2020;20:1840-8.

45. Varghese J, Malleeswaran S, Patcha RV, et al. A multicentric experience on living donor liver transplantation in COVID-19 hotspots in India. Liver Transpl. 2020. https://doi.org/10.1002/lt.25957.

46. Haugen CE, McAdams-DeMarco M, Verna EC, et al. Association between liver transplant wait-list mortality and frailty based on body mass index. JAMA Surg. 2019;154:1103-9.

47. Wiesner R, Edwards E, Freeman R, et al. Model for end-stage liver disease score and allocation of donor livers. Gastroenterol. 2003;124:91-6.

48. Samuel D, Coilly A. Management of patients with liver diseases on the waiting list for transplantation: a major impact to the success of liver transplantation. BMC Med. 2018;16:113.

49. American Society of Transplantation. 2020. https://www.myast. org/covid-19-information. Accessed 15 Nov 2020.

50. American Society of Transplant Surgeons. 2020. https://asts.org/ advocacy/covid-19-resources/asts-covid-19-strike-force/re-engag ing-organ-transplantation-in-the-covid-19-era\#.X29pqBSSk2w. Accessed 15 Nov 2020 
51. Trapani S, Masiero L, Puoti F, et al. Incidence and outcome of SARS-CoV-2 infection on solid organ transplantation recipients: a nationwide population-based study. Am J Transplant. 2020. https://doi.org/10.1111/ajt.16428.

52. Kates OS, Haydel BM, Florman SS, et al. COVID-19 in solid organ transplant: a multi-center cohort study. Clin Infect Dis. 2020. https://doi.org/10.1093/cid/ciaa1097.

53. Belli LS, Fondevila C, Cortesi PA, et al. Protective role of tacrolimus, deleterious role of age and comorbidities in liver transplant recipients with Covid-19: results from the ELITA/ELTR multicenter European study. Gastroenterology. 2020. https://doi.org/10. 1053/j.gastro.2020.11.045.

54. Raja MA, Mendoza MA, Villavicencio A, et al. COVID-19 in solid organ transplant recipients: a systematic review and metaanalysis of current literature. Transplant Rev. 2020;35:100588.

55. Fix OK, Hameed B, Fontana RJ, et al. Clinical best practice advice for hepatology and liver transplant providers during the COVID19 pandemic: AASLD expert panel consensus statement. Hepatology. 2020;72:287-304.

56. Moletta L, Pierobon ES, Capovilla G, et al. International guidelines and recommendations for surgery during Covid-19 pandemic: a systematic review. Int J Surg. 2020;79:180-8.

57. Qadan M, Hong TS, Tanabe KK, et al. A multidisciplinary team approach for triage of elective cancer surgery at the Massachusetts General Hospital during the novel coronavirus COVID-19 outbreak. Ann Surg. 2020;272:e20-1.

58. Ross SW, Lauer CW, Miles WS, et al. Maximizing the calm before the storm: tiered surgical response plan for novel coronavirus (COVID-19). J Am Coll Surg. 2020;230:1080-91.e3.

59. Morales-Conde S, Balla A, Álvarez Gallego M, et al. A dynamic scale for surgical activity (DYSSA) stratification during the COVID-19 pandemic. Br J Surg. 2020;107:e425-6.

60. Prachand VN, Milner R, Angelos P, et al. Medically necessary, time-sensitive procedures: scoring system to ethically and efficiently manage resource scarcity and provider risk during the COVID-19 pandemic. J Am Coll Surg. 2020;231:281-8.

61. NHS, clinical guide to surgical prioritisation during the coronavirus pandemic. 2020. https://www.england.nhs.uk/coronavirus/ wp-content/uploads/sites/52/2020/03/C0221-specialty-guide-surgi cal-prioritisation-v1.pdf. Accessed 25 Apr 2020.

62. Cancer patient management during the COVID-19 pandemic, ESMO. 2020. https://www.esmo.org/guidelines/cancer-patientmanagement-during-the-covid-19-pandemic. Accessed $23 \mathrm{Apr}$ 2020.

63. Saigal S, Gupta S, Sudhindran S, et al. Liver transplantation and COVID-19 (Coronavirus) infection: guidelines of the liver transplant society of India (LTSI). Hepatol Int. 2020;14:429-31.
64. Arezzo A, Francis N, Mintz Y, et al. EAES recommendations for recovery plan in minimally invasive surgery amid COVID-19 pandemic. Surg Endosc. 2021;35:1-17.

65. Puylaert CAJ, Scheijmans JCG, Borgstein ABJ, et al. Yield of screening for COVID-19 in asymptomatic patients before elective or emergency surgery using chest CT and RT-PCR (SCOUT): multicenter study. Ann Surg. 2020;272:919-24.

66. COVIDSurg Collaborative. Global guidance for surgical care during the COVID-19 pandemic. Br J Surg. 2020;107:1097-103.

67. Borgstein ABJ, Scheijmans JCG, Puylaert CAJ, et al. Yield of adding chest $\mathrm{CT}$ to abdominal CT to detect COVID-19 in patients presenting with acute gastrointestinal symptoms (SCOUT-3): multicenter study. Ann Surg. 2020. https://doi.org/10.1097/SLA. 0000000000004678.

68. Liu YL, Ren J, Yuan JP, et al. Postoperative onset and detection of SARS-CoV-2 in surgically resected specimens From gastrointestinal cancer patients with pre/asymptomatic COVID-19. Ann Surg. 2020;272:e321-8.

69. Di Maira T, Berenguer M. COVID-19 and liver transplantation. Nat Rev Gastroenterol Hepatol. 2020;17:526-8.

70. Kute V, Guleria S, Prakash J, et al. NOTTO transplant specific guidelines with reference to COVID-19. Indian J Nephrol. 2020;30:215-20.

71. Challine A, Dousset B, de'Angelis N, et al. Impact of coronavirus disease 2019 (COVID-19) lockdown on in-hospital mortality and surgical activity in elective digestive resections: a nationwide cohort analysis. Surgery. 2021. https://doi.org/10.1016/j.surg. 2020.12.036.

72. Jonker PKC, van der Plas WY, Steinkamp PJ, et al. Perioperative SARS-CoV-2 infections increase mortality, pulmonary complications, and thromboembolic events: a Dutch, multicenter, matchedcohort clinical study. Surgery. 2021;169:264-74.

73. Dumortier J, Duvoux C, Roux O, et al. Covid-19 in liver transplant recipients: the French SOT COVID registry. Clin Res Hepatol Gastroenterol. 2021;45:101639.

74. Webb GJ, Marjot T, Cook JA, et al. Outcomes following SARSCoV-2 infection in liver transplant recipients: an international registry study. Lancet Gastroenterol Hepatol. 2020;5:1008-16.

Publisher's Note Springer Nature remains neutral with regard to jurisdictional claims in published maps and institutional affiliations. 